Instituto Internacional de Investigación y Desarrollo Tecnológico Educativo INDTEC, C.A.

DOI: https://doi.org/10.29394/Scientific.issn.2542-2987.2021.6.21.2.40-57

OAl-PMH: http://www.indteca.com/ojs/index.php/Revista Scientific/oai

Artículo Original / Original Article

\title{
Potencialidad de Biocombustibles a partir de Residuos Orgánicos
}

\author{
Autores: Catherine Estefanía Chávez Altamirano \\ Universidad Estatal de Bolívar, UEB \\ catchavez@mailes.ueb.edu.ec \\ Guaranda, Ecuador \\ https://orcid.org/0000-0002-0307-4289 \\ Fernanda Galud López Calvopiña \\ Universidad Estatal de Bolívar, UEB \\ fernlopez@mailes.ueb.edu.ec \\ Guaranda, Ecuador \\ https://orcid.org/0000-0002-6821-3278 \\ Ximena Marianela Palate Chicaiza \\ Universidad Estatal de Bolívar, UEB \\ xpalate@mailes.ueb.edu.ec \\ Guaranda, Ecuador \\ https://orcid.org/0000-0001-7025-8451 \\ Carlos Rodrigo Jacome Pilco \\ Universidad Estatal de Bolívar, UEB \\ cjacome@ueb.edu.ec \\ Guaranda, Ecuador \\ https://orcid.org/0000-0002-9713-0228
}

\section{Resumen}

El propósito del estudio de revisión bibliográfica es analizar el biocombustible obtenido a partir de residuos orgánicos como parte de la alternativa verde para ayudar al medio ambiente, la biotecnología brinda diferentes formas de generar energía renovable, entre las que destaca la producción de bioetanol. El proceso de obtención del biocombustible es la etapa de fermentación del alcohol, que es una reacción biológica que se lleva a cabo en ausencia de aire, que convierte los azúcares en alcohol $\left(\mathrm{C}_{2} \mathrm{H}_{6} \mathrm{O}\right)$ y dióxido de carbono $\left(\mathrm{CO}_{2}\right)$. El desarrollo de los combustibles fósiles es la base de la sociedad, siempre que sean fuentes de energía renovables obtenidas a partir de residuos orgánicos, lo que menciona al banano, fuente importante para la producción de bioetanol, por contener un alto contenido en carbohidratos. Los biocombustibles son una fuente de progreso a nivel industrial y agrícola, y una opción energética de origen renovables y la reducción de gases efecto invernadero que se da por de la descomposición de desechos orgánicos la cual provoca que sea una prometedora opción para la suplencia de combustibles fósiles para la valorización energética de residuos orgánicos en áreas despobladas, urbanas y agroindustriales.

Palabras clave: biomasa; lignocelulósica; energía renovable; biocombustible.

Código de clasificación internacional: 2306.90 - Química de productos naturales orgánicos.

Cómo citar este artículo:

Vera, A., \& Moreira, T. (2021). Entorno familiar de personas con discapacidad: una intervención desde el Trabajo Social. Revista Scientific, 6(21), 21-39, e-ISSN: 2542-2987. Recuperado de: https://doi.org/10.29394/Scientific.issn.2542-2987.2021.6.21.1.21-39

Fecha de Recepción: 18-03-2021
Fecha de Aceptación:

03-07-2021
Fecha de Publicación: 05-08-2021 
OAI-PMH: http://www.indteca.com/ojs/index.php/Revista Scientific/oai

\title{
Artículo Original / Original Article
}

\section{Potential of Biofuels from Organic Waste}

\begin{abstract}
The purpose of the bibliographic review study is to analyze the biofuel obtained from organic waste as part of the green alternative to help the environment, biotechnology offers different ways of generating renewable energy, among which the production of bioethanol stands out. The process for obtaining biofuel is the alcohol fermentation stage, which is a biological reaction that takes place in the absence of air, which converts sugars into alcohol $\left(\mathrm{C}_{2} \mathrm{H}_{6} \mathrm{O}\right)$ and carbon dioxide $\left(\mathrm{CO}_{2}\right)$. The development of fossil fuels is the foundation of society, as long as they are renewable energy sources obtained from organic waste, which mentions bananas, an important source for the production of bioethanol, as they contain a high content of carbohydrates. Biofuels are a source of progress at an industrial and agricultural level, and an energy option of renewable origin and the reduction of greenhouse gases that occurs due to the decomposition of organic waste, which makes it a promising option for the replacement of fuels. fossils for the energy recovery of organic waste in unpopulated, urban and agro-industrial areas.
\end{abstract}

Keywords: biomass; lignocellulosic; renewable energy; biofuel. International classification code: 2306.90 - Organic natural products chemistry.

\footnotetext{
How to cite this article:

Vera, A., \& Moreira, T. (2021). Family environment of people with disabilities: an intervention from Social Work. Revista Scientific, 6(21), 21-39, e-ISSN: 2542-2987. Recovered from: https://doi.org/10.29394/Scientific.issn.2542-2987.2021.6.21.1.21-39
}

Date Received: 18-03-2021
Date Acceptance:

03-07-2021
Date Publication: 05-08-2021 


\section{Introducción}

Los residuos orgánicos provenientes de distintas superficies tanto industriales, comerciales, y de vivienda, son materias primas que tiene varias proporciones de celulosa, hemicelulosa, lignina, numerosas indagaciones realizadas otorgan a entender que se utilizan con el propósito de hacer procesos para la obtención de biocombustibles dirigidos al desarrollo de nuevas fuentes energéticas, más adelante enfocar a que se potencialicen estos recursos.

En la actualidad, Japa (2015): expone que la implementación de procesos biotecnológicos para la obtención de bienes y servicios se ha modificado en una enorme disyuntiva que conlleva resoluciones a inconvenientes derivados de la aplicación de tecnologías usuales.

Según Llenque-Díaz, Quintana, Torres y Segura (2020): al generar los biocombustibles que se generan de diferentes materias orgánicas provenientes de procesos con seres vivos, sus desperdicios permanecen siendo extensamente estudiados ya hace numerosas décadas. Las materias primas con más visión de generar bioetanol son la lignocelulósica y en los desperdicios de plantas agroindustriales son el segundo conjunto que aporta en la producción de bioetanol.

El presente trabajo tiene como objetivo investigar la obtención de los biocombustibles a partir de los residuos orgánico, la potencialidad al desarrollo de los nuevos campos de la biotecnología toda la información está basada en fuentes confiables de diversos estudios que se han realizado por los diferentes autores.

\section{Metodología}

El presente documento fue redactado mediante una revisión bibliografía a partir de bases de datos como SciELO, Dialnet, Redalyc, entre otras, también la información fue obtenida de repositorios de universidades, con el propósito 
de encontrar información relevante sobre la potencialidad de biocombustibles a partir de residuos orgánicos, donde se utilizaron palabras claves: bioetanol, residuos orgánicos, potencialidad, energía renovable, bioenergía, proceso fermentativo, hidrolisis y composición química. La selección de estos artículos fue acorde al objetivo planteado.

\subsection{Residuos orgánicos}

De acuerdo con Ministerio del Ambiente y Agua (2020a): se entiende como desechos orgánicos, todo aquello que se genera de procedencia vegetal y animal, siendo susceptible a degradarse biológicamente. Además, son las materias que proceden de trabajos de fabricación y consumo que no alcanzó ningún costo monetario.

En este sentido, Navarro, Moral, Gómez y Mataix (1995): dicen que las considerables y diversas posibilidades que ofrecen los residuos orgánicos, tienen la posibilidad de asegurar, sin caer en equivocadas expectativas, que después muy unido se podrán sugerir recursos derivados de los residuos, para cada tipo de cultivo o suelo que queremos recuperar en ello está la labor y el fervor de diversos investigadores y detrás de ella, posiblemente venga el fin conocida y política encaminadas a favorecer no solamente la investigación, sino el aprovechamiento plenamente de dichos residuales.

\subsection{Cascara de plátano}

Para Martínez-Cardozo, Cayón-Salinas y Ligarreto-Moreno (2016): la cáscara de plátano está formada de celulosa, hemicelulosa y lignina. Su distribución varía según el origen de las materias primas. También es una fuente importante de materias primas de celulosa. Es un ingrediente fuera de los plátanos, que representa alrededor del $40 \%$ del peso. argumentaron que utilizaron $5 \mathrm{~kg}$ de cáscaras de plátano, que primero deben estar limpias.

El pretratamiento se basa en reducir la capacidad de lignina y reducir la 
cristalinidad celulosa. Primero, se muele la cáscara de plátano, luego se sumerge en hidróxido de sodio satisfactoriamente, $\mathrm{NaOH} 0.1 \mathrm{~N}$ durante 15 minutos, luego se agrega el sulfato de calcio $\left(\mathrm{CaSO}_{4}\right)$, dejándolo reposar durante 3 horas y esperando a que se filtre. La hidrólisis se lleva a cabo en el reactor de presión a $(15 \mathrm{psi})$ y temperatura $\left(125^{\circ} \mathrm{C}\right)$ para obtener fármacos de glucosa.

La fermentación se lleva a cabo en un reactor, añadiendo glucosa y agregando levadura (Saccharomyces cerevisiae). Después de la fermentación, se tamiza para eliminar los materiales duros y luego se destila en una torre de destilación. El paso final es pasar el destilado por un proceso de filtros de arena o resina para obtener $95 \%$ de alcohol. El cuadro 1, muestra el análisis de algunas propiedades físicas y químicas de las cáscaras de banano de varios estudios.

Cuadro 1. Caracterización fisicoquímica de la cascara de plátano.

\begin{tabular}{|c|c|c|c|}
\hline & Experimental & Teórico & Referencia \\
\hline \multicolumn{4}{|c|}{ Análisis proximal - base seca } \\
\hline $\begin{array}{l}\text { Humedad total - Hwt, } \\
\%\end{array}$ & $84.94 \pm 0.10$ & 87.17 & \multirow{5}{*}{$\begin{array}{c}\text { Plátano verde clon Hartón } \\
\text { Común }\end{array}$} \\
\hline Material volátil- MV, \% & $74.00 \pm 1.76$ & --- & \\
\hline Ceniza-Cz, \% & $8.18 \pm 0.43$ & 7.20 & \\
\hline Carbono fijo- CF, \% & $17.82 \pm 1.18$ & --- & \\
\hline MV/CF & $4.15 \pm 0.85$ & --- & \\
\hline Poder calórico, MJ/kg & $17.39 \pm 1.27$ & 16.82 & Biomasa \\
\hline \multicolumn{4}{|c|}{ Análisis elemental, base seca libre de ceniza } \\
\hline $\begin{array}{l}\text { Humedad al aire - } \\
\text { Hwt, \% }\end{array}$ & $15.06 \pm 0.05$ & --- & \multirow{8}{*}{$\begin{array}{l}\text { Biomasas residuales } \\
\text { provenientes de cultivos } \\
\text { tradicionales en Colombia }\end{array}$} \\
\hline Carbono - C, \% & $48.40 \pm 0.21$ & 42.50 & \\
\hline Hidrogeno- $\mathrm{H}, \%$ & $7.27 \pm 0.01$ & 4.29 & \\
\hline Nitrógeno- N, \% & $1.36 \pm 0.01$ & 0.74 & \\
\hline Azufre - S, \% & $0.06 \pm 0.001$ & 0.02 & \\
\hline Oxigeno- $\mathrm{O}, \%$ & $42.91 \pm 0.18$ & 52.45 & \\
\hline $\mathrm{H} / \mathrm{C}$, base seca & $1.80 \pm 0.01$ & $1.21^{*}$ & \\
\hline $\mathrm{O} / \mathrm{C}$, base seca & $0.66 \pm 0.003$ & $0.67^{*}$ & \\
\hline \multicolumn{4}{|c|}{ Análisis estructural y bioactivo } \\
\hline Celulosa, \% & $42.87 \pm 0.23^{* *}$ & 20.90 & \multirow{3}{*}{ Residuos lignocelulósicos } \\
\hline Hemicelulosa, \% & $29.09 \pm 0.43^{* *}$ & 7.92 & \\
\hline Lignina, \% & $10.91 \pm 0.01^{* *}$ & 18.11 & \\
\hline
\end{tabular}


OAI-PMH: http://www.indteca.com/ojs/index.php/Revista Scientific/oai

Artículo Original / Original Article

\begin{tabular}{|c|c|c|c|}
\hline Extractivos totales, \% & $45.61 \pm 0.31$ & --- & \\
\hline $\begin{array}{l}\text { Fenoles totales, mg } \\
\mathrm{AC} / \mathrm{g}\end{array}$ & $15.4 \pm 0.80$ & 16.00 & \multirow{2}{*}{$\begin{array}{c}\text { Residuos industrias } \\
\text { transformadoras de frutas } \\
\text { tropicales }\end{array}$} \\
\hline $\begin{array}{l}\text { Capacidad } \\
\text { antioxidante } \\
\text { (mg Trolox/100g p.s) }\end{array}$ & $161.10 \pm 50$ & $173.10 \pm 65$ & \\
\hline
\end{tabular}

Fuente: Retto (2019).

\subsection{Cascara de arroz}

Señalando a Prada y Cortés (2010): las cáscaras de arroz son crujientes, de textura rugosa y varían en tono puede de color marrón rojizo e inclusive morado oscuro. Su consistencia disminuye, por lo que ocupa cuantioso espacio al almacenarlo. De acuerdo con el análisis de simulación de Capdevila, Kafarov, Gely y Pagano (2015): sobre el desarrollo de la fermentación del bioetanol obtenido a partir del residuo de arroz, se seleccionó el escenario de cambiador de proporción biomasa: agua de 1: 2,89; el caudal de biomasa de 50 toneladas/ hora y el ambiente de $30^{\circ} \mathrm{C}$. La temperatura de entrada del separador maximiza el rendimiento del bioetanol.

Estos cambios, hace que la realización de bioetanol sea de 8,81 toneladas/hora y la pureza sea de $65,51 \%$ peso/peso, lo que equivale a un caudal de biomasa de 50 toneladas/hora para el tratamiento de hidrólisis. Este trabajo representa un avance en el modelo de desarrollo completo en el proceso de bioetanol de generación segunda a partir de restos industriales de arroz. Consulte el cuadro 2, para ver los datos de la constitución química de la cáscara y el residuo de arroz.

Cuadro 2. Estructura química de la cáscara del cereal.

\begin{tabular}{cccc}
\hline \multicolumn{2}{c}{ Cascarilla de Arroz } & \multicolumn{2}{c}{ Ceniza de la cascarilla de arroz } \\
Componente & $\%$ & Componente & $\%$ \\
Carbono & 39,1 & Ceniza de Sílice $\left(\mathrm{SiO}_{2}\right)$ & 94,1 \\
Hidrogeno & 5,2 & Oxido de Calcio $(\mathrm{Ca} O)$ & 0,55 \\
Nitrógeno & 0,6 & Oxido de magnesio $(\mathrm{MgO})$ & 0,95 \\
Oxigeno & 37,2 & Oxido de Potasio $\left(\mathrm{K}_{2} \mathrm{O}\right)$ & 2,10 \\
Azufre & 0,1 & Oxido de Sodio $\left(\mathrm{Na}_{2} \mathrm{O}\right)$ & 0,11 \\
\hline
\end{tabular}


OAI-PMH: http://www.indteca.com/ojs/index.php/Revista Scientific/oai

\begin{tabular}{cccc}
\hline Cenizas & 17,8 & Sulfato & 0,06 \\
& & Cloro & 0,05 \\
& & Oxido de titanio $\left(\mathrm{TiO}_{2}\right)$ & 0,05 \\
& & Oxido de Aluminio $\left(\mathrm{Al}_{2} \mathrm{O}_{3}\right)$ & 0,12 \\
& & Otros componentes $\left(\mathrm{P}_{2} \mathrm{O}_{5}, \mathrm{~F}_{2} \mathrm{O}_{3}\right)$ & 1,82 \\
Total & 100,0 & & 100,0 \\
\hline
\end{tabular}

Fuente: Prada y Cortés (2010).

\subsection{Caña de azúcar}

Interpretando a Pernalete, Piña, Suárez, Ferrer y Aiello (2008): el bagazo es rico en energía; no obstante, gracias a su compleja estructura, son difíciles de digerir una vez que se usan como alimento para rumiantes, por lo cual requieren ser procesados para minimizar o inhibir la barrera física y química, y la trasparencia de la celulosa, el conjunto acetilo de hemicelulosa y el enlace de la hemicelulosa y la lignina.

En este mismo orden de ideas, para Zumalacárregui-De, Pérez-Ones, Rodríguez-Ramos, Zumalacárregui-De y Lombardi (2015): el residuo adusto contiene un $50 \%$ de celulosa, $30 \%$ de hemicelulosa y $20 \%$ de lignina. Al reportar composición elemental aproximada, se puede considerar el valor de carbono: $48,3 \%$; alto: $6 \%$; oxigeno; $43,3 \%$; y cenizas: $2,4 \%$.

\subsection{Residuo de piña}

Interpretando a Cruz, Mendoza, Chávez, Rivera y Cruz (2011): el objetivo es obtener celulosa y bioetanol a partir de la cáscara de piña (residuos agrícolas). El uso de cáscara evitará la ingestión de siembras, evitará el uso inadecuado de la tierra y el uso de restos vegetales agropecuarios e industriales. Asimismo, los productos obtenidos tienen valor añadido y pueden convertirse en beneficios. Es por ello que se estudia el proceso para eliminar el bagazo de piña y conseguir glucosa mediante hidrólisis ácida de celulosa y corteza.

La glucosa neutraliza a pH 5 y se fermenta en mediano anaeróbico, 
tratando la bacteria Saccharomyces cerevisiae, diferentes períodos de fermentación tales como; 36, 40, 48 y 72 horas y su temple se mantiene a 30 ${ }^{\circ} \mathrm{C}$. La tasa de conversión de la celulosa obtenida es del $60 \%$ y la Espectrometría Infrarroja con Transformada de Fourier (FTIR) confirma que la citológica es de tipo II. El bioetanol se obtiene por destilación, el rendimiento del bagazo es del $35 \%$ y el rendimiento del bagazo es del $57 \%$ con celulosa con un lapso de fermentación de 48 y 72 h, equitativamente.

La celulosa extraída del bagazo de piña seca, demostró una fachada porosa y poco dúctil, y una coloración crema, La transformación obtenida ha sido del $60 \%$, ya que de 5 gramos de bagazo de piña se han conseguido 3 gramos de celulosa. El examen de FTIR se realizó con diferentes estudios para lograr una celulosa de varias materias primas y lo más importante es clasificar el tipo de celulosa que se tiene. Se presenta que ha sido viable lograr celulosa del bagazo de piña y por medio de los análisis de FTIR, se corroboraron los equipos propios de la celulosa, la cual ha sido celulosa de tipo II (hidrolisis de conversión a glucosa).

\subsection{Origen de los residuos}

El Ministerio del Ambiente y Agua (2020b): presenta que los restos orgánicos se refieren a una imponente variedad de restos que se originan naturalmente a lo extenso del ciclo vital, como consecuencia de las funcionalidades orgánicas de sustento y persistencia o son producto del aprovechamiento por el ser humano de los recursos bióticos.

\subsection{Potencial de los residuos orgánicos en América latina}

Por su parte, Razo, Ludeña, Saucedo, Astete-Miller, Hepp y Vildósola (2007): añaden que la implementación de residuos para desarrollar biocombustibles en ciertos sitios se aprecia con monumental exceptiva pues esto nos asiste a reducir la contaminación que son elaboradas por industrias, 
agrícolas etcétera. El realizar tecnologías enfocadas a fomentar la obra de nuevas alternativas sustentables.

La fuente potencial de elaboración de bioetanol está la caña de azúcar, de hecho, en todos los países de América Latina y el Caribe, existe el exceso de oferta pues es generalizado. Además, la producción de maíz, trigo y sorgo se concentra en Argentina, que tiene una gran cantidad de producción de granos y una ventaja comparativa. Los países considerados pueden producir un total de 20 mil millones de litros de bioetanol anualmente a partir del excedente de expedición, de los cuales el $58 \%$ se destina a la sacarosa, el $22 \%$ al maíz y al trigo el $18 \%$. Siendo exactamente igual al $26 \%$ del porcentaje de mezcla de consumo de gasolina promedio regional actual.

\subsection{Beneficio de desechos agroindustriales para la mejora de la eficacia del ambiente}

En consideración a Soto (2012), citado por Vargas y Pérez (2018a): cualquier residuo agrícola industrial tiene posibilidad para originar "bioenergía a partir de biocombustibles como la biomasa energética, el bioetanol, el biodiésel, entre otros, e incluyen un rango amplio de productos que a su vez se han dividido en diferentes tipos de combustible" (pág. 63). El aprovechamiento de estos residuos se transforma y se convierte en un tema de importancia por los beneficios que trae al medio ambiente y aportes al sector económico. Estos son utilizados como una oportunidad de mejorar al medio ambiente ya que la contaminación de suelos y de fuentes hídricas va en constante aumento a medida que transcurre el tiempo son difíciles reconstruirse.

Con relación a eso, Valdés y Palacios (2016), citados por Vargas y Pérez (2018b): aseveran que "en general los biocombustibles son considerados como una estrategia energética más sustentable que la quema de combustibles fósiles, que genera emisiones de gases de efecto invernadero 
y contribuye al cambio climático" (pág. 63); un combustible obtenido a partir de residuos industriales disminuye los gases que emana de los combustibles fósiles, aportando al mantenimiento de un ecosistema más sano y limpio.

Igualmente detalla Chiroque (2018): que los biocombustibles se consiguen de biomasa o sea de "[...] cualquier tipo de materia orgánica que haya tenido su origen inmediato en el proceso biológico de organismos recientemente vivos, como plantas, o sus deshechos metabólicos (el estiércol) [...]" (pág. 18). La obtención de biocombustibles también se puede realizar a partir de los desechos provenientes de los animales, debido a que posee un alto contenido de nutrientes que ayudan en el desarrollo y crecimiento de microorganismos para la producción de bioetanol. En el cálculo de obtención del bioetanol se usa el método descrito en la figura 1.

Figura 1. Flujograma - Producción de bioetanol a partir de biomasa.

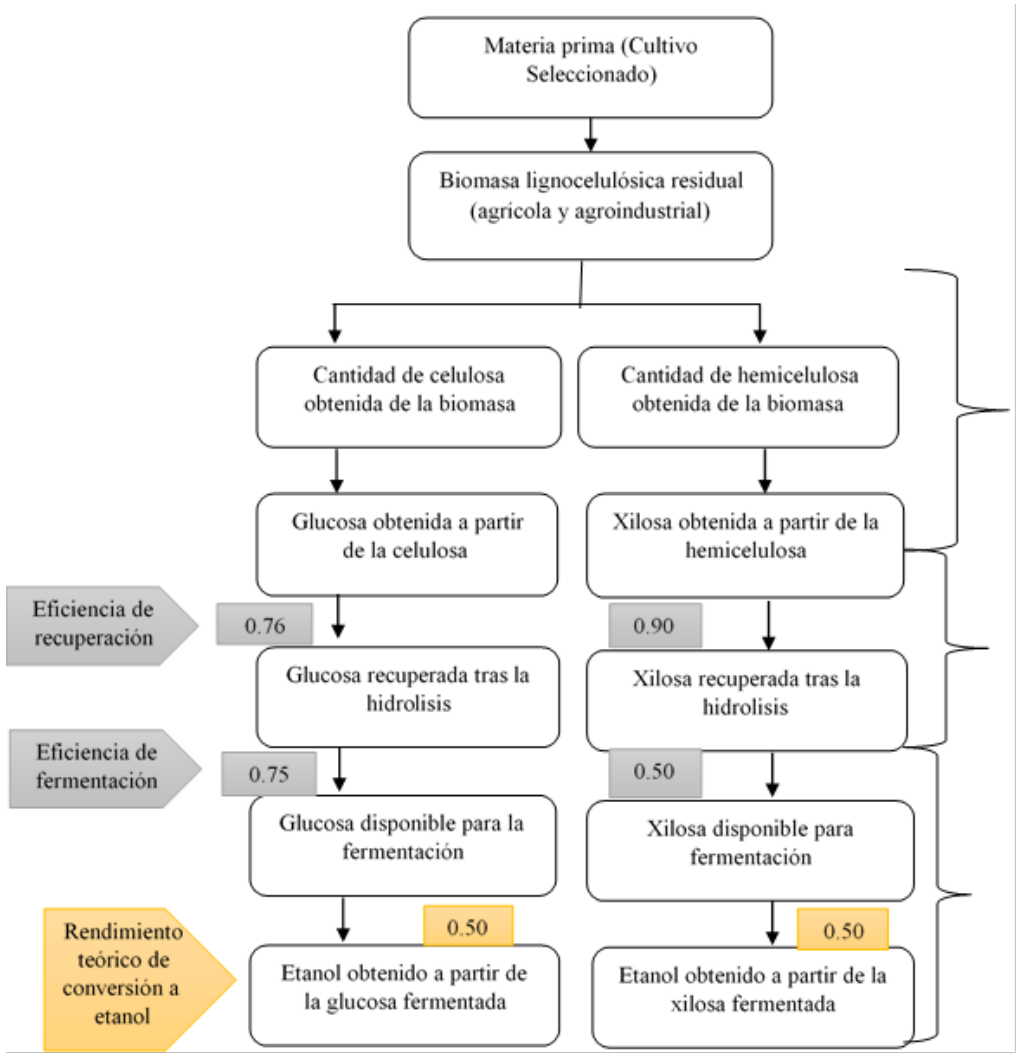

Fuente: Retto (2019). 


\section{Resultados}

Según los estudios realizados para la obtención de biocombustibles, utilizando como materias primas residuos orgánicos, es necesario que estos insumos primarios contengan elevados porcentajes de hidratos de carbono principalmente en forma de azucares reductores o que a su vez puedan ser hidrolizables mediante métodos físicos o enzimáticos.

De la información analizada, encontramos que los investigadores Cruz, Mendoza, Chávez, Rivera y Cruz (2011): utilizando residuos de la piña, sometidos a hidrolisis, alcanzaron un $57 \%$ en la fermentación durante un periodo de tiempo de 48 a 72 horas a temperatura de $30^{\circ} \mathrm{C}$, como mencionan Martínez-Cardozo, Cayón-Salinas y Ligarreto-Moreno (2016): mediante hidrolisis se obtuvo un rendimiento aproximado de 95 por ciento su proceso duro un tiempo de 15 minutos, una vez añadido el sulfato de calcio se deja en reposo de tres horas para su filtración, que de acuerdo con Prada y Cortez (2010): indican que el desarrollo fermentativo para la obtención de bioetanol mediante tratamiento de hidrolisis, presenta un rendimiento del $65,51 \%$ equivalente a 50 toneladas de biomasa, mostrando una alternativa para el desarrollo de combustibles.

\section{Conclusiones}

El conocimiento y tecnologías desarrolladas hasta la fecha, respecto al uso de residuos agroindustriales para la producción de bioenergía, muestran ser altamente eficientes, lo que garantiza una producción sostenible de biocombustibles a partir de biomasa residual, contribuyendo de esta manera al desarrollo agroindustrial y a la disminución de la contaminación ambiental por el tratamiento no adecuado de este tipo de residuos.

La urgente necesidad en el mundo, de contar con diversas fuentes de energía, sin lugar a duda debe también estar orientada a la explotación de la inmensa potencialidad de la biomasa residual desde donde se puede producir 
bioenergía, con el fin de sustituir en parte la gran demanda de combustibles fósiles.

\section{Referencia}

Capdevila, V., Kafarov, V., Gely, C., \& Pagano, A. (2015). Simulación del proceso fermentativo para la obtención de bioetanol a partir de residuos de arroz. Avances en Ciencias e Ingeniería, 6(2), 11-21, eISSN: 0718-8706. Recuperado de:

https://www.redalyc.org/articulo.oa?id=323639772002

Chiroque, J. (2018). Capacidad de producción de bioetanol a través de la degradación de residuos sólidos orgánicos en Chiriaco, 2018. Tesis. Chiclayo, Perú: Universidad de Lambayeque. Recuperado de: https://repositorio.udl.edu.pe/handle/UDL/175

Cruz, R., Mendoza, A., Chávez, M., Rivera, J., \& Cruz, M. (2011). Aprovechamiento del bagazo de piña para obtener celulosa $y$ bioetanol. Afinidad, 68(551), 38-43, e-ISSN: 2339-9686. Recuperado de: https://raco.cat/index.php/afinidad/article/view/267945

Japa, J. (2015). Hidrólisis enzimática de biomasa celulosa obtenida de la cáscara de banano maduro mediante Trichoderma sp. para la obtención de bioetanol. Trabajo de Titulación. El Oro, Ecuador: Universidad Técnica de Machala. Recuperado de:

http://repositorio.utmachala.edu.ec/handle/48000/2824

Llenque-Díaz, L., Quintana, A., Torres, L., \& Segura, R. (2020). Producción de bioetanol a partir de residuos orgánicos vegetales. REBIOL: Revista de Investigación Científica, 40(1), 21-29, e-ISSN: 2313-3171. Recuperado de:

https://revistas.unitru.edu.pe/index.php/facccbiol/article/view/2991 Martínez-Cardozo, C., Cayón-Salinas, G., \& Ligarreto-Moreno, G. (2016). 
OAI-PMH: http://www.indteca.com/ojs/index.php/Revista Scientific/oai

Artículo Original / Original Article

genotipos de plátano y banano. Ciencia y Tecnología Agropecuaria, 17(2), 217-227, e-ISSN: 2500-5308. Recuperado de: https://doi.org/10.21930/rcta.vol17 num2 art:491

Ministerio del Ambiente y Agua (2020a,b). Manual de aprovechamiento de residuos orgánicos municipales. Primera Edición, ISBN: 978-99428846-0-2. Quito, Ecuador: Asociación de Municipalidades del Ecuador y la Fundación ACRA.

Navarro, J., Moral, R., Gómez, I., \& Mataix, J. (1995). Residuos orgánicos y agricultura. ISBN: 84-7908-194-5. España: Universidad de Alicante.

Pernalete, Z., Piña, F., Suárez, M., Ferrer, A., \& Aiello, C. (2008). Fraccionamiento del bagazo de caña de azúcar mediante tratamiento amoniacal: efecto de la humedad del bagazo y la carga de amoníaco. Bioagro, 20(1), 3-10, e-ISSN: 1316-3361. Recuperado de: https://www.redalyc.org/articulo.oa?id=85720101

Prada, A., \& Cortés, C. (2010). La descomposición térmica de la cascarilla de arroz: una alternativa de aprovechamiento integral. Orinoquia, 14(1), 155-170, e-ISSN: 0121-3709. Recuperado de:

https://www.redalyc. org/articulo.oa?id=89622691013

Razo, C., Ludeña, C., Saucedo, A., Astete-Miller, S., Hepp, J., \& Vildósola, A. (2007). Producción de biomasa para biocombustibles líquidos: el potencial de América Latina y el Caribe. ISBN: 978-92-1323127-2. Santiago de Chile: Naciones Unidas.

Retto, P. (2019). Potencial energético de la producción de bioetanol a partir de residuos agroindustriales lignocelulósicos en el Perú. Tesis. Trujillo, Perú: Universidad Nacional de Trujillo. Recuperado de: https://dspace.unitru.edu.pe/handle/UNITRU/12153

Vargas, Y., \& Pérez, I. (2018a,b). Aprovechamiento de residuos agroindustriales para el mejoramiento de la calidad del ambiente. Revista Facultad de Ciencias Básicas, 14(1), 59-72, e-ISSN: 1900- 
4699. Recuperado de: https://doi.org/10.18359/rfcb.3108

Zumalacárregui-De, L., Pérez-Ones, O., Rodríguez-Ramos, P., Zumalacárregui-De, B., \& Lombardi, G. (2015). Potencialidades del bagazo para la obtención de etanol frente a la generación de electricidad. Ingeniería Investigación y Tecnología, 16(3), 407-418, eISSN: 2594-0732. Recuperado de:

http://dx.doi.org/10.1016/j.riit.2015.05.001 


\section{Catherine Estefanía Chávez Altamirano e-mail: catchavez@mailes.ueb.edu.ec}

Nacida en la provincia de Pichincha, Ciudad de Quito,

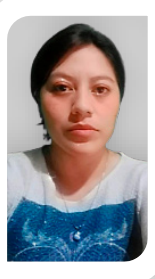
Ecuador, el 11 de enero del año 1996. Los estudios secundarios los realice en el Colegio General Pintag en la especialidad de Ciencias Generales, posteriormente los estudios superiores los realice en el Instituto Superior Tecnológico Luis A. Martinez (Agronómico) (ISTLAMA), obteniendo el título de Tecnólogo en Agroindustrias; actualmente asesora en publicidad de productos alimenticios, destacándome en el área comercial. 
OAI-PMH: http://www.indteca.com/ojs/index.php/Revista Scientific/oai

Artículo Original / Original Article

\section{Fernanda Galud López Calvopiña \\ e-mail: fernlopez@mailes.ueb.edu.ec}

Nacida en Provincia Orellana, Cantón Loreto, Ecuador, el

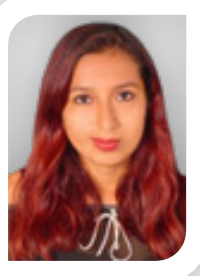

14 de marzo del año 1997. Tecnóloga en Agroindustria del Instituto Tecnológico Superior Luis A. Martínez (Agronómico) (ISTLAMA); Obtuve certificación de buenas prácticas de manufactura, asistente técnica en la asociación de productores agropecuarios la paz. 
OAI-PMH: http://www.indteca.com/ojs/index.php/Revista Scientific/oai

Artículo Original / Original Article

\section{Ximena Marianela Palate Chicaiza}

e-mail: xpalate@mailes.ueb.edu.ec

Nacida en la provincia de Chimborazo, Cantón Guano,

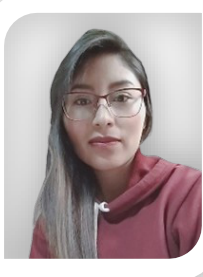

Ecuador, el 24 de junio del año 1996. Los estudios secundarios los realice en la Unidad Educativa "17 de abril" en la especialidad de Ciencias Generales; posteriormente los estudios superiores los realice en el Instituto Superior Tecnológico Luis A. Martinez (Agronómico) (ISTLAMA), obteniendo el título de Tecnólogo en Agroindustrias. 


\section{Carlos Rodrigo Jacome Pilco \\ e-mail: cjacome@ueb.edu.ec}

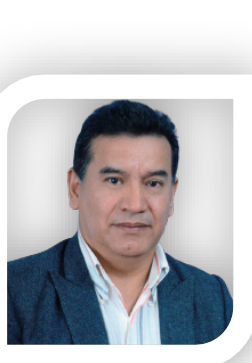

Nacido en la ciudad de Guaranda, provincia de Bolívar, Ecuador, el 20 de julio de 1961. Doctor en Ciencias (PhD.), en la especialidad de Biotecnología por el Centro de Investigación y de Estudios Avanzados del Instituto Politécnico Nacional (CINVESTAV-IPN), México; 19 años como profesor-investigador titular en la Universidad Estatal de Bolívar (UEB), en el área de Biotecnología Agroindustrial; autor y coautor de varios artículos científicos y participante en diferentes proyectos de investigación con fondos nacionales e internacionales.

El contenido de este manuscrito se difunde bajo una Licencia de Creative Commons ReconocimientoNoComercial-Compartirlgual 4.0 Internacional 